\title{
Do Vulnerável Ser ao Resiliente Envelhecer: Revisão de Literatura
}

\author{
Carlos António Sampaio de Jesus Laranjeira ${ }^{1}$ \\ Escola Superior de Saúde - Campus Universitário Jean Piaget de Viseu
}

\begin{abstract}
RESUMO - Desde o dealbar da infância até à velhice, a resiliência edifica-se por meio de um jogo complexo de processos defensivos de ordem intrapsíquica e de fatores de proteção internos e externos. O objetivo deste estudo foi investigar a produção científica em periódicos indexados nas bases de dados Medline, Lilacs e PsycINFO sobre o conceito de resiliência, no período de 1994-2004, e analisar a contribuição dessa literatura na especificidade do indivíduo idoso. Trata-se de um estudo documental e retrospectivo que evidenciou quatro núcleos temáticos: precursores da resiliência, resiliência e envelhecimento, relatividade dos fatores de proteção e resiliência e velhice bem sucedida. A análise da literatura permite constatar um incremento nas publicações, sendo ainda notória a falta de assertividade quanto à perenidade da resiliência ao longo do desenvolvimento humano, com especial enfoque no envelhecimento.
\end{abstract}

Palavras-chave: resiliência; idoso; envelhecimento; pesquisa; vulnerabilidade.

\section{From Vulnerable Being to Resilient Ageing: Review of the Literature}

\begin{abstract}
From the dawn of childhood into old age, resilience is built up through a complex interplay of defensive processes of an intra-psychic nature, together with internal and external protective factors. The objective of this study was to investigate scientific findings on the concept of resilience, published in reviews indexed by the Medline, Lilacs and PsycINFO databases between 1994 and 2004, analysing the contribution made by this literature in the specific field of old age. This is a documental, retrospective study which brings out four thematic nuclei: predecessors of resilience; resilience and ageing; the relative nature of protection factors; resilience and successful ageing. On the basis of this analysis of the literature, it is possible to discern an increase in the volume of publications. Nevertheless, it remains true that there is a failure to assert the enduringness of resilience throughout human development, in particular in terms of old age.
\end{abstract}

Key words: resilience; old age; ageing; research; vulnerability.

Os transtornos do comportamento e, eventualmente, as psicopatologias surgem como um dos resultados das relações familiares gravemente carenciadas ou do confronto com acontecimentos de vida traumáticos (Whitbourne, 2001).

Em face desses contextos, os profissionais da saúde e os diferentes agentes socioeducativos tentam tradicionalmente compreender os fatores de risco e de vulnerabilidade, a fim de ajudar os indivíduos, avaliando os riscos posteriores de morbidade. Todavia, a análise das reações dos indivíduos confrontados com a adversidade revela a multiplicidade de respostas adaptativas e vislumbra que essa questão não pode ficar circunscrita a uma abordagem centrada na vulnerabilidade e na inadaptação (Greenfield \& Marks, 2004; Inui, 2003). Essa complexidade é atestada pela idiossincrasia comportamental dos indivíduos e a variedade do seu crescimento psíquico. É certo que alguns indivíduos podem revelar perturbações sociais, escolares, familiares que não devem ser negligenciadas, no entanto outros, em contextos igualmente desfavoráveis, não apresentam perturbações comportamentais ou psíquicas e fazem prova de formas de ajustamento aparentemente inexplicáveis.

1 Endereço: Escola Superior de Saúde do Campus Universitário Jean Piaget de Viseu, Estrada do Alto do Gaio, Galifonge, Lordosa, Viseu, Portugal 3515-776.E-mail: carloslaranjeira@ hotmail.com
É aí, então, que surge o conceito de resiliência, que implica adaptação em face do perigo, a capacidade de sair vencedor de uma prova que poderia ter sido traumática, com uma força renovada. Uma polarização exclusiva nos aspectos vulneráveis dos indivíduos e nos fatores de risco do seu contexto de vida pode levar a que se negligencie os contributos do estudo dos sujeitos que demonstram procedimentos adaptativos e de recursos psíquicos que os preservam dos aspectos psicotóxicos do seu ambiente (Fonagy, 1994). As pesquisas sobre a resiliência denotam equívocos nos estudos teóricos e preditivos centrados num dissecar dos aspectos vulneráveis dos indivíduos e dos fatores de risco, negligenciando as suas competências e fatores de proteção, ante acontecimentos ameaçadores.

Perante a complexidade dos ângulos de abordagem da resiliência enquanto processo multidimensional, cada vez mais os estudos se debruçam sobre critérios particulares ligados ao tema ou referem-se a formas de expressão da resiliência estudadas em função dos diferentes contextos, interno ou externo aos sujeitos. Assim, designações variadas se desenvolvem sobre as manifestações da resiliência em função da idade dos sujeitos, do contexto ambiental, afetivo, social e cultural...

Porém, e apesar do entusiasmo dos últimos anos, a resiliência no idoso continua difícil de precisar (Wagnild, 2003) na medida em que a maioria das investigações está relacionada com o estudo da resiliência infantil e preocupam-se particularmente com os aspectos precoces que a constituem. 
Com o discernimento de redirecionar o pensamento e a prática sobre os contornos teóricos que sustentam a concepção de resiliência, o estudo objetivou investigar a produção científica sobre o conceito de resiliência, analisando a contribuição dessa literatura na especificidade do indivíduo idoso.

\section{Método}

Trata-se de uma pesquisa retrospectiva, descritiva e documental que teve como recorte temporal o período entre 1994 e 2004. A coleta dos dados integrou periódicos indexados nas bases de dados Medline, Lilacs e PsycINFO. Esses periódicos divulgam a ciência, são de carácter multidisciplinar, contribuindo para a construção da complexidade do saber. A inclusão dos artigos obedeceu a critérios de presença de pelo menos um dos descritores - adaptação, vulnerabilidade, modelo da resiliência, idoso, acontecimentos de vida - previamente seleccionados no texto e contexto do trabalho. A coleta de dados foi realizada no período de novembro de 2004 a maio de 2005, tendo-se extraído dos periódicos selecionados 44 artigos sobre a temática, os quais representam a amostra deste estudo.

Para a organização das informações contidas nas publicações científicas encontradas foram seguidos os passos preconizados por Minayo (1993), leitura flutuante de todos os artigos, mediante uma pesquisa exploratória procurando identificar o objeto, os objetivos do estudo e os resultados do mesmo, posteriormente os dados foram registrados sob a forma de fichas de leitura.

Para análise dos dados deste estudo utilizou-se a técnica de Análise de Conteúdo, proposta por Bardin (1979). A Análise de Conteúdo na sua história mais recente, isso é, enquanto técnica de tratamento de dados considerada cientificamente, procura a sua lógica na interpretação cifrada de material de natureza qualitativa. Conseqüentemente, trata-se de uma técnica de descrição objetiva, sistemática e quantitativa do conteúdo manifestado nas comunicações tendo por fim interpretá-las. Os conteúdos temáticos encontrados nos resumos dos trabalhos foram categorizados segundo quatro núcleos temáticos: Precursores da resiliência, Resiliência e envelhecimento, Relatividade dos fatores de proteção e Resiliência e velhice bem sucedida.

\section{Resultados e Discussão}

Da análise dos 44 artigos que versam sobre a temática da resiliência no idoso, são dissecados os resultados obtidos conforme os núcleos temáticos identificados.

\section{Precursores da resiliência}

Abordando a emergência do conceito de resiliência, foram identificadas sete publicações que situam a resiliência numa encruzilhada epistemológica, integrando contributos das teorias psicanalíticas e comportamentais e que impregna as abordagens clínicas e socioeducativas contemporâneas. Consoante as investigações e as tentativas de aplicação no terreno, assim a resiliência é abordada tanto como um conceito, um traço de carácter, como o resultado de um processo, o próprio processo ou um modo específico de funcionamento (comportamental ou psíquico).

A resiliência é assim apresentada como um fenômeno, um funcionamento, ou ainda, por vezes, uma arte de se adaptar às situações adversas (condições biológicas e sociopsicológicas) desenvolvendo capacidades ligadas aos recursos internos (intrapsíquicos) e externos (ambiente social e afetivo), que permitem aliar uma construção psíquica adequada à inserção social. A emergência desse conceito surgiu nos países anglo-saxônicos e norte-americanos, e podemos citar, como precursores dessa abordagem, investigadores como Haggerty (1996) e Fonagy (1994, 2001), mas também francófanos como Cyrulnik $(1999,2001)$ ou Manciaux $(1999,2001)$. Desde esses primeiros trabalhos sobre resiliência, podemos constatar uma evolução significativa desse modelo teórico que conseguiu se desenvolver e consolidar os seus contornos teóricos estando posto de parte do empobrecimento desse conceito, de se aplicar a tudo e a todos, de se tornar um simples sinônimo do conceito de coping (estratégia de ajustamento) ou ainda do de adaptação (Tugade, Fredrickson $\&$ Barrett, 2004).

Podemos realçar a abundância na literatura de definições latas da resiliência, como a de Fonagy (1994), para quem a resiliência pode ser considerada como um desenvolvimento normal perante circunstâncias difíceis. Esse autor relaciona a resiliência com as teorias da vinculação, ao passo que o psicanalista Lemay (citado por Andreoletti \& Lachman, 2004) propõe uma abordagem bastante transversal da resiliência, estabelece antes a ligação com a teoria psicanalítica, na qual os processos defensivos usados pelos indivíduos têm muitas vezes origem em percursos comportamentais e psíquicos aparentemente patológicos. A resiliência refere-se portanto a um processo complexo que resulta da interação entre o indivíduo e o seu meio ambiente.

O conceito de resiliência depressa ganhou adeptos no domínio social, comportamental e cognitivo, mais recentemente foi enriquecido no contato com a psicopatologia (Jang, Poon \& Martin, 2004). Parece ter encontrado hoje os seus títulos de nobreza ao tornar-se não num simples conceito, porém num modelo de compreensão do sujeito humano em todas as fases do seu desenvolvimento, na sua dimensão normal e patológica.

\section{Resiliência e envelhecimento}

Os primeiros trabalhos sobre resiliência dizem respeito sobretudo às crianças e aos adolescentes, todavia prolongamse agora ao estudo das diferentes fases de vida: desde a infância até à velhice.

A população de idosos tornou-se nomeadamente um dos grupos de investigação mais instrutivos, foram 18 as pesquisas que preconizam o estudo da plasticidade resiliente dos sujeitos em relação ao desenvolvimento e ao envelhecimento, segundo uma perspectiva que engloba a vida inteira. Se é habitual falar da força psicológica nas crianças e jovens (Fonagy, 1994; 2001), pode-se questionar sobre o que isso poderá significar no idoso; por outras palavras, poder-se-ia falar de resiliência e velhice? Traumatismos de todos os tipos podem advir com o avançar da idade, os falecimentos (do cônjuge, de familiares, de amigos) tornam-se cada vez mais frequen- 
tes. A perda da utilidade social e a doença podem ocasionar perturbações graves nos idosos. Assim, o envelhecimento poderia ser considerado como um contexto de risco, contudo, os idosos reagem de formas muito diferentes (Whitbourne, 2001). Alguns parecem desenvolver um comportamento que facilmente poderemos classificar como resiliente, no qual os acontecimentos normais e esperados de vida são sobretudo precipitantes de novas expressividades ao invés de ameaças à continuidade do self (Sullivan \& Fisher, 1994).

Aguerre (2002) não hesita em afirmar que a definição muito generalista, que faz referência à resiliência das crianças como a capacidade de ultrapassar a adversidade graças aos seus recursos psicológicos, pode se aplicar igualmente aos idosos que conseguem viver bem apesar das dificuldades ligadas à idade com que se deparam. Nessa óptica, a resiliência pode ser abordada como um funcionamento estabelecido desde longa data, eventualmente desde a infância. A abordagem da resiliência como processo confere-lhe uma característica dinâmica e evolutiva inscrita na temporalidade e portanto não fixa, logo não perene. Assim, há investigações que visam estabelecer a perenidade (ou não) da resiliência, adoptando uma abordagem retrospectiva de maneira a analisar o percurso dos idosos considerados resilientes. O estudo da resiliência no idoso pode dar lugar também às investigações transgeracionais (a transmissão da resiliência) e sobre o impacto geracional do funcionamento resiliente de um progenitor em relação à sua descendência (Aguerre, 2002; De Tychey, 2001).

Contudo, inúmeras questões permanecem. Os centenários, que começam a ser estudados devido à crescente esperança média de vida nas sociedades ocidentais, fazem parte das investigações sobre a resiliência e o envelhecimento. Entre as questões que se colocam, encontramos as que questionam a própria longevidade. Assim, será que a longevidade é um critério que ateste a resiliência, ou então que seja resultado de um funcionamento resiliente? Diferentes perspectivas estão sendo aplicadas nas pesquisas com abordagens multifatoriais sobre a população dos idosos e a resiliência. Essas investigações certamente que poderão produzir conhecimentos importantes para completar a abordagem teórica do modelo da resiliência e eventualmente modular as práticas.

\section{Relatividade dos fatores de proteção}

Procurando examinar os fatores de proteção, no quadro da resiliência do idoso, que reduzem o efeito do risco e as reações negativas, foram registradas 10 publicações. Nessa óptica, Whitbourne (2001), a partir da observação de famílias desfavorecidas, conclui que a resiliência assenta em três tipos de fatores de proteção. Segundo esse autor, a tríade da força psicológica da resiliência é composta por: fatores individuais (o temperamento, a reflexão, as capacidades cognitivas, sentimentos de empatia, humor e as competências sociais), fatores familiares (o calor humano, a coesão e a atenção por parte do principal prestador de cuidados) e fatores de suporte (rede de apoio familiar, o sistema de serviços sociais). Segundo Fonagy (2001), esses três pólos de proteção favorecem a resiliência porque melhoram a auto-estima e a auto-eficácia e abrem novas possibilidades para o sujeito. Porém, é necessário utilizar as noções de risco e de proteção com prudência e abordá-las de forma interdisciplinar. Com efeito, a prática clínica mostra que certos fatores considerados como protetores e que contribuem para a resiliência, podem, em doses elevadas, ter um efeito contrário. Tomemos como exemplo a auto-estima, fator de resiliência que encontramos no centro da maior parte das abordagens. Um mínimo de auto-estima é necessário e útil para formar a resiliência. No entanto, o seu desenvolvimento exagerado pode levar a personalidades marcadas pela arrogância, pelo desprezo dos outros e das leis, o que irá prejudicar a adaptação, tornando-se mesmo um fator de risco, situação que irá contrariar o funcionamento resiliente (Greenfield \& Marks, 2004). O carácter protetor ou de risco de um fator depende, sobretudo, do contexto qualitativo relacional em que se desenvolve. É o caso, por exemplo, do apoio familiar ou do suporte social que constituem fatores de proteção importantes para os sujeitos. Ora, no caso do abandono e da negligência do idoso, é justamente no quadro familiar e relacional do indivíduo idoso que o perigo se situa (Manciaux, 2001). Assim, a família e a rede relacional mais alargada tanto podem funcionar como fatores de proteção, como, pelo contrário, o próprio lugar dos fatores de risco.

Procurando definir um "retrato robô" do indivíduo resiliente independentemente da idade e do sexo, segundo Cyrulnik (1998), o indivíduo resiliente seria um sujeito com um coeficiente de inteligência elevado, capaz de ser autônomo e eficaz nas relações com o meio, ter boas capacidades de adaptação relacional e empatia, capaz de prever e planificar e finalmente ter sentido de humor. Claro está que a totalidade dessas características não é garantia de um bom funcionamento resiliente, mas esses diferentes elementos encontram-se em muitos dos sujeitos considerados resilientes. Cyrulnik explora com essa abordagem características essencialmente de ordem cognitiva, comportamental e psicossocial, colocando de fora a abordagem metapsicológica dos processos psíquicos que intervêm na resiliência, isso é, os mecanismos de defesa e adaptação, a sua mentalização e posterior comunicação sob a forma de imagens e emoções sentidas, condição que segundo De Tychey (2001) é essencial para o funcionamento resiliente a longo prazo.

\section{Resiliência e velhice bem sucedida}

A ciência conseguiu a proeza inédita de aumentar, espetacularmente, a duração média de vida. Mas o que ela não esclareceu é como viver esse suplemento de vida. Efetivamente, mais tempo para viver não é, necessariamente, melhor tempo para viver. Implícitos a essa temática encontraram-se cinco artigos que colocam o problema do envelhecimento bem sucedido.

Para o estudo da resiliência dos idosos, um dos critérios que na maior parte das vezes é ressaltado nas análises é o da velhice bem sucedida. Os pesquisadores propõem diferentes índices que aliam critérios objetivos (saúde, longevidade, capacidades cognitivas) a critérios subjetivos avaliados pelo próprio idoso (sensação de bem-estar, satisfação) (Inui, 2003).

A inserção social é tida em conta de forma diferente, assim os partidários do "descomprometimento" consideram como critério suplementar a aceitação do desinvestimento e a renúncia a certos objetivos devido a involução ligada à 


\section{A. S. J. Laranjeira}

idade; ao passo que outros teóricos consideram como critério o estabelecimento de novos investimentos e relações interpessoais (Schweitzer \& Quintard, 2001).

Assim, para Rowe e Kahn (1999), uma velhice bem sucedida, aliada a um percurso resiliente, significa a ausência de doença ou pelo menos de invalidez (ou ausência de handicap), um elevado nível de funcionamento mental e psicológico e a inserção social por meio de atividades e relações interpessoais. Diferentes critérios são evocados pelos investigadores, como, por exemplo, a crise de identidade estruturante (na linha de Erikson), que sugere uma abordagem bipartida, a geratividade versus estagnação, ou seja, o envelhecimento bem sucedido é medido não apenas pela ausência de problemas, mas por indicadores de bem estar subjetivo, como são a satisfação com a vida, a felicidade, a moral, o contentamento, a qualidade de vida percebida ou outras medidas relacionadas negativamente como a depressão e a ansiedade (Wagnild, 2003). De fato, algumas pesquisas sobre a resiliência no idoso preocupam-se com o estudo de alguns traços de personalidade assim como as formas de adaptação e com as estratégias de ajustamento, e tentam especificar os modos de funcionamento que seriam característicos dos idosos considerados resilientes.

Ao passo que outros teóricos enfocam conceitos bastante difíceis de estudar, como a sagacidade, apresentada como a faculdade própria do idoso ou pelo menos que se desenvolveria com a idade madura, nesse contexto, a sagacidade seria definida como a tendência para abordar positivamente as dificuldades da vida (Schmitt, 2004). A abordagem da velhice permanece sujeita por vezes às extrapolações não destituídas de idealismo e/ou estereótipos culturais que introduzem enviesamentos de que é preciso precaver-se nas pesquisas.

\section{Considerações Finais}

Os trabalhos encontrados sobre resiliência tentam conciliar uma pluralidade de abordagens teóricas e de domínios de intervenção clínica. Diferentes disciplinas conexas contribuíram para o seu desenvolvimento e usufruem das suas aplicações. Quer se trate da Psiquiatria, da Psicologia, da Sociologia, da Prevenção Social ou da Psicopatologia.

O núcleo central da resiliência é o fato de trabalhar os potenciais e os modos dos sujeitos em face do perigo. Por outras palavras, de ter em conta os fatores de proteção e não apenas os fatores de risco. A abordagem mais divulgada na nossa sociedade é influenciada pelo modelo psicopatológico e da vulnerabilidade. O modelo da resiliência, contudo, não deve contrapor-se a este, mas tem de ser considerado como uma forma complementar de avaliar a vulnerabilidade dos sujeitos.

A vantagem do modelo da resiliência pode ser comprovada na medida em que ele permite compreender o desenvolvimento de sujeitos que sofreram contextos traumáticos graves e que, no entanto, se desvencilham. Ele fornece, nomeadamente, elementos para a compreensão das estratégias socializadas que alguns sujeitos descobrem, apesar de um contexto familiar de início incapaz.

Na compreensão do fenômeno da resiliência questionamos igualmente os seus fracassos e revelamos os seus limites.
O conceito de resiliência pode ser considerado como uma resposta complementar à abordagem da vulnerabilidade, mas não corresponde por isso à idéia de invencibilidade ou insensibilidade, remete, pelo contrário, para a idéia de flexibilidade e de adaptação. Se considerarmos que a resiliência se baseia nas experiências relacionais precoces e na possibilidade de estabelecer laços sociais posteriores, isso confere-lhe um carácter variável, consoante os indivíduos e num mesmo indivíduo, ao longo do seu desenvolvimento. Os trabalhos encontrados sobre o devir dos sujeitos resilientes deveriam nos permitir completar a compreensão e a abordagem do processo resiliente questionando a sua perenidade assim como o seu funcionamento; todavia, muitas questões precisam de ser exploradas: É preciso pensar na resiliência como um potencial presente em todos os sujeitos? É possível agir e estimular a resiliência nos sujeitos, famílias, nos grupos sociais? Qual é a formação de que necessitam os profissionais que intervêm e quais os limites das suas competências?

O processo psíquico e comportamental do indivíduo resiliente perante uma situação traumatogénica seria comparável ao trabalho da ostra que, para se proteger do grão de areia que a fere segregando nácar à volta do intruso, vai arredondar as asperezas do grão de areia e dar origem a uma jóia preciosa (Cyrulnik, 1999). A resiliência seria forjada pelo indivíduo perante as agressões da vida e o sujeito resiliente conservaria, assim, esse precioso potencial, que o iria ajudar a enfrentar a sua trajetória de vida em boas condições. A metáfora da ostra perlífera ilustra bem como, por vezes, é a partir de uma experiência de sofrimento que podemos revelar forças até aí mantidas latentes e desconhecidas. Nas práticas clínicas, o risco será de subestimar os aspectos de eventual sofrimento ligado ao desencadear de processos defensivos nos sujeitos considerados resilientes e de considerar erradamente a sua resiliência como invulnerabilidade perante os contextos nocivos. A resiliência surge assim como resultado de um processo paradoxal no qual o confronto com o traumatismo e a ferida geram criatividade. Todavia, a beleza da metáfora da ostra perlífera dita que o indivíduo resiliente permanece um ser que foi ferido, cuja ferida não está completamente cicatrizada e pode estar mascarada pelo comportamento resiliente.

\section{Referências}

Aguerre, C. (2002). Quels sont les facteurs psychologiques garants d'une vieillesse réussie? Pratiques psychologiques, 1, 15-27.

Andreoletti C. \& Lachman M. (2004). Susceptibility and resilience to memory aging stereotypes: education matters more than age. Experimental Aging Research, 30(2), 129-148.

Bardin L. (1979). Análise de Conteúdo. Lisboa: Edições 70.

Cyrulnik, B. (1998). Ces enfants qui tiennent le coup. Revigny-surOmain: Hommes et Perspectives.

Cyrulnik, B. (1999). Un merveilleux malheur. Paris: Odile Jacob. Cyrulnik, B. (2001). Les vilains petits canards. Paris: Odile Jacob.

De Tychey, C. (2001). Surmonter l'adversité: les fondements dynamiques de la résilience. Cahiers de Psychologie Clinique, 16(1), 49-68.

Fonagy, P. (1994). The theory and practice of resilience. Journal of Child Psychology and Psychiatry, 35(2), 231-257. 
Fonagy, P. (2001). Développement de la psychopathologie de l'enfance à l'âge adulte. Psychiatrie de L'Enfant, 44(2), 333369.

Greenfield E. \& Marks N. (2004). Formal volunteering as a protective factor for older adults' psychological well-being. Journal of Gerontology: Social Sciences, 59(5), S258-S264.

Haggerty, R. (1996). Stress, Risk and Resilience in Children and Adolescents. Cambridge: University Press.

Inui, T. (2003). The need for an integrated biopsychosocial approach to research on successful aging. Annual of International Medicine, 139(5), 391-394.

Jang Y., Poon L. \& Martin P. (2004). Individual differences in the effects of disease and disability on depressive symptoms: the role of age and subjective health. International Journal of Aging and Human Development, 59(2), 125-137.

Manciaux, M. (1999). La résilience: mythe ou réalité? Em M. Gabel (Org.), Maltraitance psychologique (pp. 99-119). Paris: Fleurus.

Manciaux, M. (2001). La résilience: résister et se construire. Genebra: Cahiers médico-sociaux.

Minayo, M. (1993). O desafio do conhecimento: pesquisa qualitativa em saúde. Rio de Janeiro: Hucitec-Abrasco.

Rowe, J. \& Kahn, R. (1999). Successful aging. New York: Dell Publishing.
Schmitt E. (2004). Active ageing, decreasing capability, social disadvantage and age stereotypes: a contribution to resilience and vulnerability in older adulthood. Zeitschrift für Gerontologie und Geriatrie, 37(4), 280-292.

Schweitzer, M. \& Quintard, B. (2001). Personalité et maladies: Stress, coping et ajustement. Paris: Dunod.

Sullivan, W. \& Fisher, B. (1994). Intervening for success: Strengthsbased case management and successful aging. Journal of Gerontological Social Work, 22, 61-74.

Tugade M., Fredrickson B. \& Barrett L. (2004). Psychological resilience and positive emotional granularity: examining the benefits of positive emotions on coping and health. Journal of Personality, 72(6), 1161-90.

Wagnild, G. (2003). Resilience and successful aging. Comparison among low and high income older adults. Journal of Gerontological Nursing, 29(12), 42-49.

Whitbourne, S. (2001). Adult development and aging: Biopsychosocial perspectives. New York: John Wiley and Sons.

Recebido em 02.03.2006

Primeira decisão editorial em 27.03.2006

Versão final em 10.01.2007

Aceito em 12.03.2007 